\title{
Astronomical Tasks for Tests of X-Ray Optics in VZLUSAT-1 Nanosatellite
}

\author{
Martin Blazek, Petr Pata, Adolf Inneman, and Petr Skala \\ Department of Radioelectronics, Faculty of Electrical Engineering, Czech Technical University in Prague, Technicka 2, \\ Prague 6, Czech Republic \\ Correspondence should be addressed to Martin Blazek; martin.blazek@fel.cvut.cz
}

Received 13 September 2016; Revised 31 December 2016; Accepted 16 January 2017; Published 7 February 2017

Academic Editor: Valery Nakariakov

Copyright (C) 2017 Martin Blazek et al. This is an open access article distributed under the Creative Commons Attribution License, which permits unrestricted use, distribution, and reproduction in any medium, provided the original work is properly cited.

\begin{abstract}
VZLUSAT-1 nanosatellite (scheduled launch in spring 2017 from India) is a CubeSat mission which, besides other instrumentation, contains X-ray desk to perform efficiency tests of the X-ray optics. In this article the analysis of potential observational candidates for VZLUSAT-1 X-ray board is presented together with the suggestion of observational modes, laboratory measurements, and estimations of exposure settings.
\end{abstract}

\section{Introduction}

VZLUSAT-1 is a nanosatellite developed in VZLU (http:// www.vzlu.cz/) Institute in Prague (Czech Republic) and its launch is scheduled for April 2017 from India. Its architecture corresponds to CubeSat missions with the size of two standard units [1]. The goals of this nanosatellite include meteorological and radiation measurements, composite material experiments, and tests of X-ray optics in the space environment. X-ray board consists of "Lobster eye" optics [2], which is to be tested in space, two ultraviolet sensors (pointing the same direction as the X-ray optics), one infrared sensor and TimePix detector [3] working in two modes to measure either count of X-ray photons or their spectral energetic distribution. Lobster eye optics has not been tested yet in space research. To perform the efficiency tests in space environment, one can use the X-ray observations of the Sun, lunar reflections, terrestrial reflections, cosmic background, and brightest astronomical $\mathrm{X}$-ray sources.

\section{VZLUSAT-1}

VZLUSAT-1 is a two-unit nanosatellite (ca. $10 \times 10 \times 20 \mathrm{~cm}$ ) with X-ray optics having focal length $f$ of $250 \mathrm{~mm}$. TimePix detector onboard VZLUSAT- 1 has $256 \times 256$ pixels each having physical size of $55 \mu \mathrm{m}$. Total size (with side $d$ ) of the chip is $14.08 \times 14.08 \mathrm{~mm}$. For this configuration, the field of view
(FOV) is $3.2^{\circ}$ for the square side and $4.5^{\circ}$ for diagonal direction. For estimated low-orbit speed (roughly 90 minutes per orbit) of the satellite with the theoretical assumption of zero rotation the FOV would move by $4^{\circ}$ per minute. Particularly during first few weeks before the stabilization of the satellite the FOV would depend, namely, on the satellite rotation. The layout of the satellite from [1] can be seen in Figure 1.

The sensitivity of the detector is determined by the $300 \mu \mathrm{m}$ thick silicon layer. Spectral variation of such a layer in $\mathrm{X}$-ray can be simulated from the data given by National Institute of Standards and Technology (https://www.nist.gov/pml/ data/xraycoef/) based on X-ray mass attenuation coefficients [4] as can be seen in Figure 2. Highest sensitivity of complete instrument (TimePix detector $+\mathrm{X}$-ray optics) is inside the spectral range of $3-8 \mathrm{keV}$, while rapidly declining for higher energies.

Exact orbital elements are unknown until the successful launch, but the inclination of the orbit should be as close to polar orbit as possible. Because of the low-orbit position the elements would vary during a time due to the atmospheric dissipation. Electromagnetic stabilization is supposed to point the satellite along its orbiting trajectory. Webpages http://vzlusat1.cz/en/ provide images and information about technological setup and satellite itself. Corresponding scientific description can be found in [5]. The principals of astrophysical X-ray monitoring from this CubeSat mission are then explained in [6]. 


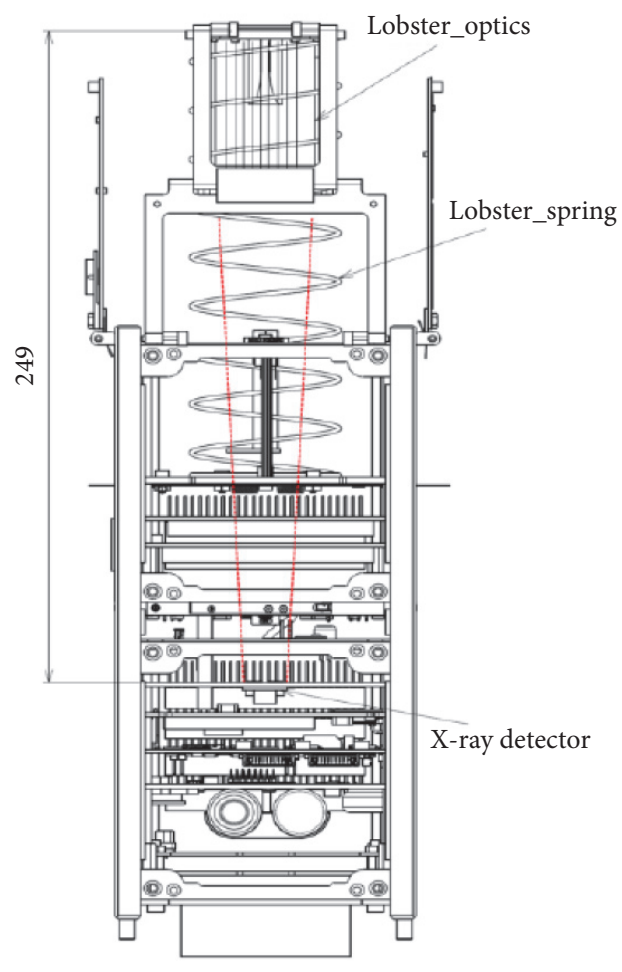

FIgURE 1: Optical layout of X-ray package inside VZLUSAT-1 satellite (image taken from [1]).

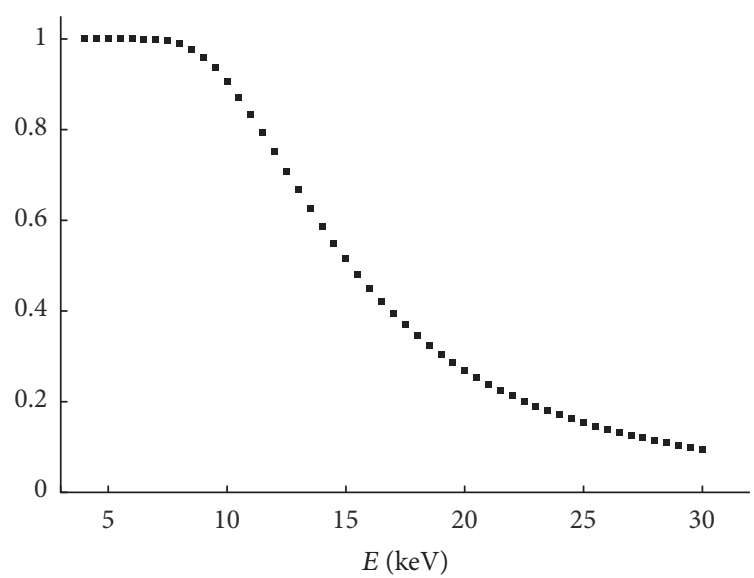

Figure 2: Relative transmission of X-ray detector onboard VZLUSAT-1 based on silicon mass attenuation coefficients.

\section{Solar Observations}

Our Sun is the brightest natural regular source of X-ray photons for our satellite. The size of the Sun $\left(0.53^{\circ}\right)$ will roughly cover one-sixth of FOV and depending on the observational angle it would pass through the FOV in 56-75 seconds. If ideally having a polar orbit and no internal rotation then the satellite would catch the Sun twice a year with six months break. Due to the larger FOV than the size of the Sun (with its ecliptical speed of $1.01^{\circ}$ per day), there might be maximal theoretical chance to catch the complete Sun in those two periods
16 times a day during 3-4 days each time roughly for 1 minute. Practically the orbital elements and rotation will be more complicated so the chance will be much lower and will need exact computations of the rotation and orbital predictions.

Due to the changes in the solar activity the X-ray flux varies. GOES-15 satellite (https://www.ngdc.noaa.gov/stp/ satellite/goes/) with one of the instruments having observational spectral range from $0.5 \AA$ to $4 \AA(3-25 \mathrm{keV})$ has very similar X-ray spectral sensitivity and therefore is the best option for calibration of the sensors and estimation of their initial settings. The solar X-ray light curve from GOES-15 satellite in 3-25 keV energy range for the period from January 2014 to May 2015 can be seen in Figure 3. The flux $F$ varies from $10^{-9}$ up to $1.8 \cdot 10^{-4} \mathrm{~W} \mathrm{~m}^{-2}$ with the mean $\bar{F}_{S}$ and median $\widetilde{F}_{S}$ values

$$
\begin{aligned}
& \bar{F}_{S}=9.8 \cdot 10^{-8} \mathrm{~W} \mathrm{~m}^{-2}, \\
& \widetilde{F}_{S}=4.3 \cdot 10^{-9} \mathrm{~W} \mathrm{~m}^{-2} .
\end{aligned}
$$

Outside several solar flare activity the X-ray intensity of the Sun in spectra range of $3-25 \mathrm{keV}$ remains in general in the degree range between $\mathrm{nW} / \mathrm{m}^{2}$ and $\mu \mathrm{W} / \mathrm{m}^{2}$.

3.1. Solar UV Hunting. For possible hunting of the solar position with fast-rotating and fast-moving low-orbit satellite one wide-field and one narrow-field ultraviolet (UV) sensor are incorporated into X-ray board. Those two sensors SG01LB18 have spectral responsivity between $231 \mathrm{~nm}$ and $309 \mathrm{~nm}$ with its peak in $280 \mathrm{~nm}$ (UVB/UVC). Effective chip area of this sensor is $1 \mathrm{~mm}^{2}$. The data (http://lasp.colorado.edu/sorce/) taken by the SORCE (Solar Radiation and Climate Experiment) [7] shows quite stable flux (in terms of degree compared to X-ray data) with the mean value $F_{\mathrm{UV}}$ at

$$
F_{\mathrm{UV}}=(17.917 \pm 0.063) \mathrm{W} \mathrm{m}^{-2}
$$

for the same period as investigated for X-ray. UV data from SORCE satellite [7] can be seen in Figure 3. Assuming perpendicular observation by UV sensor and its effective collection area the solar power caught by the sensor would be ca. $18 \mu \mathrm{W}$.

\section{Lunar Observations}

The Moon is not producing X-rays, although it is reflecting some ratio generated in the Sun. The ROSAT mission (Röntgen Satellite [8]) measured the Moon in the X-ray spectrum during the first quarter and measured the total power of the lunar X-rays published in 1991 which were $P_{M}=1.2$. $10^{12} \mathrm{erg} \mathrm{s}^{-1}$ [9]. Assuming the distance of the Moon as $D_{M}=$ $3.8 \cdot 10^{8} \mathrm{~m}$ (low-orbit is negligible) then the X-ray flux $F_{M}$ at the satellite during the Full Moon phase (multiplication factor 2) can be estimated as

$$
F_{M}=2 \cdot \frac{P_{M}}{4 \pi D_{M}^{2}} \doteq 1.3 \cdot 10^{-13} \mathrm{~W} \mathrm{~m}^{-2},
$$

which is just 4 orders lower than the median solar X-ray flux computed in previous section; hence lunar observations 


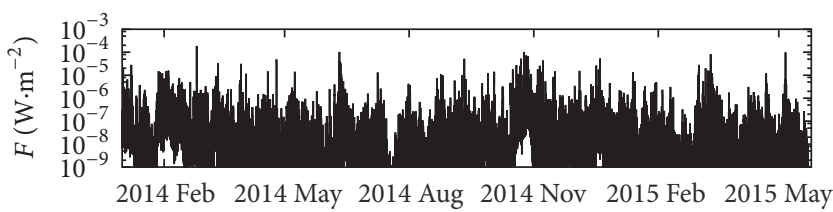

(a)

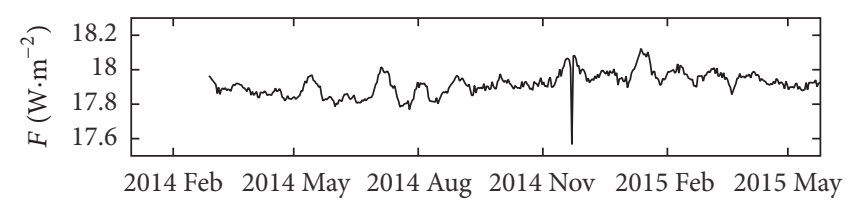

(b)

FIgURE 3: Solar X-ray observations by GOES-15 satellite in $0.5 \AA$ - $4 \AA$ spectral range (3-25 keV) in (a) and near UV observations by SORCE satellite in spectral range 231-309 nm in (b) during the period from January 2014 to May 2015.

should be configured with ten thousand time longer expositions than solar observations to provide similar signal-tonoise ratio. Although this estimation might have error of another degree as the spectral range of ROSAT mission was $0.1-2 \mathrm{keV}$.

UV detectors on board are useful for fast detection of solar presence in the field of view, although false reflection from the Moon can be caught as well. The Global Ozone Monitoring Experiment GOME [10] was launched in 1995 and was observing several terrestrial and extraterrestrial phenomena including lunar albedo in the spectral range covering near UV and visible light. For VZLUSAT-1 UV sensors it is crucial to set lower limit of positive detection to avoid confusion between the Sun and the Moon. The averaged geometrical Moon albedo measured by GOME between 1995 and 1996 in the spectral range $231-309 \mathrm{~nm}$ goes from 3 to 5 percent [11]. Assuming highest albedo of this spectral range and Full Moon reflection then the flux of the Moon in UV would be maximally

$$
F_{\mathrm{UV}}=(896 \pm 3) \mathrm{mW} \mathrm{m}^{-2} \text {, }
$$

which in the case of effective collection area of VZLUSAT-1 UV sensors correspond to the power of $0.9 \mu \mathrm{W}$.

One disadvantage of lunar observation compared to solar observation is its nonpoint spatial reflection of X-rays. Even though the instrumental optics provide one-dimensional focus of source (into line), the Moon would cover approximately one-sixth of FOV during Full Moon, which has to be taken in account during actual observation and analysis.

\section{Astronomical Observations}

Many astronomical X-ray sources are Soft X-ray Transients (SXT) which are bursts of X-ray binary systems with accreting neutron stars or black hole. Like other high energy astronomical phenomena as Gamma-ray bursts, those SXT are just occasional (often single) flares which usually last hours or days. It is highly improbable that VZLUSAT-1 could catch those sources accidentally, although some brightest galactic regular X-ray sources have to be checked if they can be potentially observational candidates. Similar X-ray spectral range to VZLUSAT-1 X-ray detector has space instrument XMM-Newton [12] (X-ray Multi-Mirror Mission) of European Space Agency, 0.2-12 keV. Table 1 shows several potential astronomical candidates for quality tests of the Lobster eye optics [2] on board VZLUSAT-1. The mean flux $\bar{F}_{X}$, median $\widetilde{F}_{X}$, maximal flux $F_{\text {max }}$, and minimal flux $F_{\text {min }}$ in Table 1 are converted from erg $\mathrm{cm}^{-2} \mathrm{~s}^{-1}$. The data in this table come from 3XMMS-DR5 catalogue (http://heasarc.gsfc .nasa.gov/W3Browse/xmm-newton/xmmssc.html) of XMMNewton project, which is extended from standard 2XMMS catalogue [13].

Outside those astronomical objects listed in Table 1, there is another X-ray source, which cannot be observed directly by XMM-Newton due to its high luminosity: Sco X-1 (or V818 Sco) [14]. This low mass X-ray binary is actually the brightest $\mathrm{X}$-ray source in the sky other than the Sun or the diffuse X-ray background radiation and estimation from XMM-Newton instrument in the range $2-10 \mathrm{keV}$ are according to [15]

$$
F_{\text {Sco }}=3.4 \cdot 10^{-8} \mathrm{erg} \mathrm{cm}^{-2} \mathrm{~s}^{-1}=3.4 \cdot 10^{-11} \mathrm{~W} \mathrm{~m}^{-2} \text {. }
$$

Older measurements from RXTE satellite (Rossi X-ray Timing Explorer) from 1999 in [16] estimate the flux in a bit wider spectral range $2-20 \mathrm{keV}$ as

$$
F_{\text {Sco }}=2.4 \cdot 10^{-7} \mathrm{erg} \mathrm{cm}^{-2} \mathrm{~s}^{-1}=2.4 \cdot 10^{-10} \mathrm{~W} \mathrm{~m}^{-2} \text {, }
$$

which is just 1-2 orders lower than the median solar flux as counted in previous sections.

\section{Calibration Procedure and Filtering}

To get the estimation of detectable fluxes of potential observational candidates, the laboratory measurements are necessary. Lobster eye optics [2] together with TimePix detector [3] was tested in X-ray tunnel with source in Titanium Xray emission of $4.5 \mathrm{keV}$. Optical module was composed of 182 glass wedges of the average thickness $150 \mu \mathrm{m}$ and 90 reflective double-sided gold-plated foils. X-ray testing was realized in the vacuum chamber at the University of Colorado at Boulder, USA. The X-ray tube with a Ti anode (energy $4.5 \mathrm{keV}$ ) was used as the source of X-rays. The source-todetector distance was $18 \mathrm{~m}$. Setup of the instrument inside the tunnel is in Figure 5.

Figure 4 shows the sum of the counts on the detector with $4.5 \mathrm{keV}$ energy for 3 different modes: darkframe including just radiation background, mode just with TimePix detector without optics, and mode with complete set including the optics. The crosses in the image represent raw measured data, while the solid line for each mode (excluding basic darkframe) represent mathematical result after filtration. 
TABLE 1: List of potential astronomical X-ray sources to be used as observational candidates for tests of X-ray optics. Data are computed from 3XMMS-DR5 catalogue of XMM-Newton mission. The measured fluxes $F @ 0.2-12 \mathrm{keV}$ in this table represent the mean $\bar{F}_{X}$, the median $\widetilde{F}_{X}$, the minimal $F_{\min }$, and the maximal value $F_{\max }$.

\begin{tabular}{|c|c|c|c|c|c|}
\hline Name & Type & $\bar{F}_{X}\left[\mathrm{~W} \mathrm{~m}^{-2}\right]$ & $\widetilde{F}_{X}\left[\mathrm{~W} \mathrm{~m}^{-2}\right]$ & $F_{\min }\left[\mathrm{W} \mathrm{m}^{-2}\right]$ & $F_{\max }\left[\mathrm{W} \mathrm{m}^{-2}\right]$ \\
\hline Mrk 421 & Blazar & $4.3 \cdot 10^{-13}$ & $3.5 \cdot 10^{-13}$ & $1.2 \cdot 10^{-18}$ & $1.2 \cdot 10^{-12}$ \\
\hline $3 \mathrm{C} 273$ & Blazar & $1.7 \cdot 10^{-13}$ & $1.6 \cdot 10^{-13}$ & $2.7 \cdot 10^{-14}$ & $2.8 \cdot 10^{-13}$ \\
\hline 1ES 1959+650 & Blazar & $1.4 \cdot 10^{-13}$ & $1.4 \cdot 10^{-13}$ & $1.4 \cdot 10^{-13}$ & $1.5 \cdot 10^{-13}$ \\
\hline Capella & Star & $9.9 \cdot 10^{-14}$ & $1.1 \cdot 10^{-13}$ & $7.1 \cdot 10^{-15}$ & $1.9 \cdot 10^{-13}$ \\
\hline V1223 Sgr & Star & $9.4 \cdot 10^{-14}$ & $9.4 \cdot 10^{-14}$ & - & - \\
\hline Mrk 501 & Blazar & $6.3 \cdot 10^{-14}$ & $6.5 \cdot 10^{-14}$ & $4.5 \cdot 10^{-14}$ & $7.9 \cdot 10^{-14}$ \\
\hline$\eta$ Car & Star & $5.6 \cdot 10^{-14}$ & $5.5 \cdot 10^{-14}$ & $3.4 \cdot 10^{-15}$ & $1.7 \cdot 10^{-13}$ \\
\hline AB Dor & Star & $5.2 \cdot 10^{-14}$ & $4.9 \cdot 10^{-14}$ & $3.8 \cdot 10^{-14}$ & $7.7 \cdot 10^{-14}$ \\
\hline V2400 Oph & Star & $4.7 \cdot 10^{-14}$ & $4.7 \cdot 10^{-14}$ & $4.4 \cdot 10^{-14}$ & $5.0 \cdot 10^{-14}$ \\
\hline FO Aqr & Star & $3.9 \cdot 10^{-14}$ & $3.9 \cdot 10^{-14}$ & - & - \\
\hline 3C 454.3 & Blazar & $2.9 \cdot 10^{-14}$ & $2.9 \cdot 10^{-14}$ & $1.2 \cdot 10^{-14}$ & $4.5 \cdot 10^{-14}$ \\
\hline EV Lac & Star & $1.8 \cdot 10^{-14}$ & $1.8 \cdot 10^{-14}$ & - & - \\
\hline V711 Tau & Star & $4.2 \cdot 10^{-14}$ & $1.6 \cdot 10^{-14}$ & $1.1 \cdot 10^{-17}$ & $1.4 \cdot 10^{-13}$ \\
\hline GK Per & Nova & $6.1 \cdot 10^{-14}$ & $1.5 \cdot 10^{-14}$ & $1.1 \cdot 10^{-14}$ & $1.6 \cdot 10^{-13}$ \\
\hline $3 C 279$ & Blazar & $1.5 \cdot 10^{-14}$ & $1.5 \cdot 10^{-14}$ & $1.5 \cdot 10^{-14}$ & $1.6 \cdot 10^{-14}$ \\
\hline BL Lac & Blazar & $1.4 \cdot 10^{-14}$ & $1.4 \cdot 10^{-14}$ & $9.4 \cdot 10^{-15}$ & $1.8 \cdot 10^{-14}$ \\
\hline S5 $0716+714$ & Blazar & $1.7 \cdot 10^{-14}$ & $1.2 \cdot 10^{-14}$ & $7.2 \cdot 10^{-15}$ & $3.7 \cdot 10^{-14}$ \\
\hline V2487 Oph & Nova & $6.5 \cdot 10^{-15}$ & $6.5 \cdot 10^{-15}$ & $5.9 \cdot 10^{-15}$ & $7.1 \cdot 10^{-15}$ \\
\hline $3 \mathrm{C} 66 \mathrm{~A}$ & Blazar & $1.5 \cdot 10^{-15}$ & $1.5 \cdot 10^{-15}$ & $1.2 \cdot 10^{-16}$ & $3.0 \cdot 10^{-15}$ \\
\hline Sgr A* & Milky Way center & $1.4 \cdot 10^{-14}$ & $1.4 \cdot 10^{-15}$ & $3.9 \cdot 10^{-17}$ & $6.9 \cdot 10^{-14}$ \\
\hline V834 Cen & Star & $7.7 \cdot 10^{-15}$ & $9.0 \cdot 10^{-16}$ & $3.4 \cdot 10^{-17}$ & $2.2 \cdot 10^{-14}$ \\
\hline M31 & Galaxy & $2.0 \cdot 10^{-15}$ & $3.5 \cdot 10^{-16}$ & $7.9 \cdot 10^{-18}$ & $1.6 \cdot 10^{-14}$ \\
\hline
\end{tabular}

TABLE 2: Count-rates $R$ and fluxes $F$ of Lobster eye optics compared to clear data without optics at $4.5 \mathrm{keV}$ Titanium X-ray emission. Raw measured data between 1 and 20 seconds and mathematically filtered data correspond to Figure 4.

\begin{tabular}{lcccc}
\hline & $R_{\text {raw }}$ & $R_{\text {filtered }}$ & $F_{\text {raw }}$ & $F_{\text {filtered }}$ \\
& \multicolumn{2}{c}{$\left[\right.$ counts $\left.\cdot \mathrm{s}^{-1}\right]$} & $\left.1^{-12} \mathrm{~W} \cdot \mathrm{m}^{-2}\right]$ \\
\hline No optics & $182 \pm 11$ & $166 \pm 19$ & $662 \pm 40$ & $604 \pm 69$ \\
Lobster eye & $27.3 \pm 4.8$ & $26.1 \pm 4.2$ & $99 \pm 18$ & $95 \pm 15$ \\
\hline
\end{tabular}

Total size of the used chip is $14.08 \times 14.08 \mathrm{~mm}$, from which the area of the detector is $A=1.9712 \cdot 10^{-4} \mathrm{~m}^{2}$. Incident flux on the detector $F_{\mathrm{LAB}}$ can be expressed as

$$
F_{\mathrm{LAB}}=\frac{n E_{0}}{T A}
$$

where $E_{0}$ is the energy of photons, $n$ their number, $T$ exposure time, and $A$ already mentioned aperture. The count-rates $R=n / T$ for these measurements can be converted to fluxes according to (7). Measured count-rates and converted fluxes for the exposures between 1 and 20 seconds (Figure 4) are both in Table 2. From this table it can be seen that the scattering on the optics reduces the total flux on the detector with the ratio $S=(15 \pm 2.7) \%$ (the ratio between the case of count-rate without and with the optics).
The space background simulation is difficult, but the nanosatellite PROBA-V [17] carried onboard similar detector (Medipix). From results we can estimate that most of the background noise would be done by heavier particles (protons, neutrons, and $\alpha$-particles). These particles produce traces in our imaging system much larger than one-pixel area. The idea of the image processing filtration for space conditions is based on keeping just one-pixel photon events with filtering out cosmic background including heavier particles (such as $\alpha$-particles or protons). For sparse events such an 8 neighbour pixel filtration works sufficiently while for denser areas made by longer exposures it filters out even hidden onepixel X-ray photon events. Due to low radio downlink rate the filtering is processed immediately on the satellite.

\section{Expected Count-Rates}

To estimate the count-rates $R$ of selected astronomical observational candidate fluxes $F$ from Figure 1, one would need photon energy $E_{M}$, TimePix spectral sensitivity $\kappa\left(E_{M}\right)$ of the instrument at this energy, effective area of the detector $A$, and the transmission coefficient of the instrument including the optics: $S=0.15 \pm 0.027$ @ $4.5 \mathrm{keV}$ (see previous section).

When assuming simplified flat spectral emissions of the candidates then the corresponding photon energies $E_{M}$ for the specific spectral range can be easily estimated as the mean value of this spectral range. Overview of analysed catalogues 


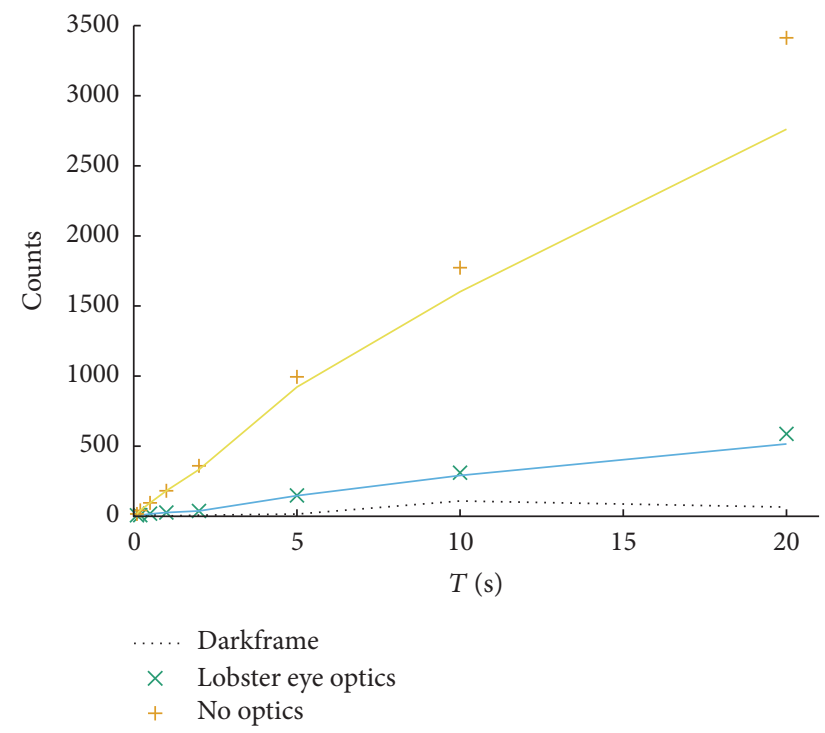

FIgURE 4: Laboratory count-rates @ $4.5 \mathrm{keV}$ Titanium X-ray emission for clear data (no optics), Lobster eye data, and closed shutter (darkframe with cosmic background). Crosses represent raw data and solid lines results after mathematical 8-neighbour one photon event filtration.

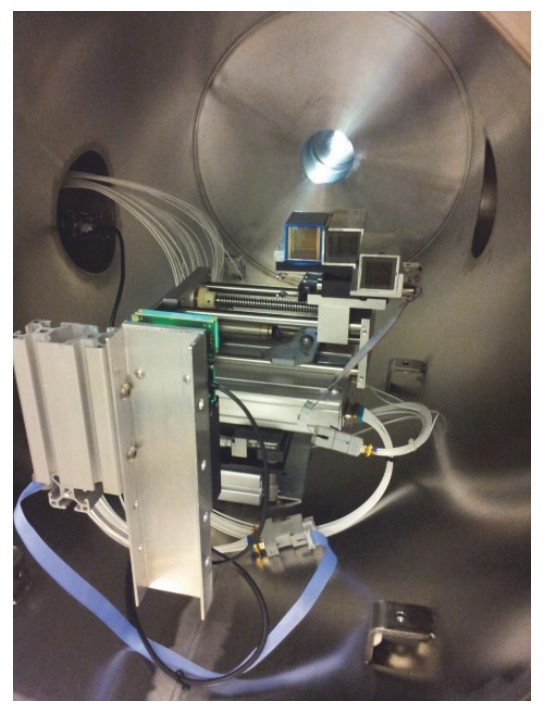

FIGURE 5: Experimental setup for calibration procedure inside vacuum chamber of X-ray optics (University of Colorado at Boulder): image taken from [1].

is in Table 3. Included are appropriate spectral ranges, mean energies $E_{M}$, and TimePix spectral sensitivities $\kappa\left(E_{M}\right)$. The estimated count-rates $R$ would be therefore

$$
R=S \cdot \kappa\left(E_{M}\right) \cdot F \frac{A}{E_{M}} .
$$

Table 4 summarizes the count-rates $R$ given by (8). Estimated fluxes for this table were calculated in previous sections, and the corresponding mean energies $E_{M}$ and TimePix spectral sensitivities $\kappa\left(E_{M}\right)$ are taken from Table 3.
TABLE 3: Mean spectral energies $E_{M}$ for selected space instruments and catalogues used in this article together with corresponding spectral sensitivity $\kappa\left(E_{M}\right)$ of TimePix detector.

\begin{tabular}{lcccc}
\hline Project catalogue & Targets & $\begin{array}{c}\text { Range } \\
{[\mathrm{keV}]}\end{array}$ & $\begin{array}{c}E_{M} \\
{[\mathrm{keV}]}\end{array}$ & $\begin{array}{c}\kappa\left(E_{M}\right) \\
{[-]}\end{array}$ \\
\hline GOES-15 & Sun & $3-25$ & 14 & 0.587 \\
ROSAT & Moon & $0.1-2$ & 1.05 & 0.999 \\
XMM-Newton & Deep sky objects & $0.2-12$ & 6.1 & 0.999 \\
RXTE & Sco X-1 & $2-20$ & 11 & 0.833 \\
\hline
\end{tabular}

TABLE 4: Estimations of count-rates per second $R$ of selected observational candidates for X-ray optics with TimePix sensor onboard VZLUSAT-1 mission. Only the observational candidates with estimated chance of catching one photon per one minute are shown; the other candidates from Table 1 can be computed from (8).

\begin{tabular}{lccc}
\hline & $\bar{R}$ & $\widetilde{R}$ & $R_{\max }$ \\
{$\left[\text { counts } s^{-1}\right]^{-1}$} & \\
\hline Sun & 76 & 33 & 1400000 \\
Full Moon & 0.023 & - & - \\
Sco X-1 (RXTE) & 3.37 & - & - \\
Sco X-1 (XMM) & 1.03 & - & - \\
Mrk 421 & 0.013 & 0.01 & 0.036 \\
\hline
\end{tabular}

In results of Table $4, \bar{R}$ is the mean count-rate per second, $\widetilde{R}$ the median value, and $R_{\max }$ the maximal value. Only the observational candidates with estimated chance of catching one photon per one minute are selected in Table 4 . The reason is in expected field view, which would move from one side to the other in approximately 50 seconds (depending on actual orbit geometry of satellite). Other candidates from Table 1 can be computed according to (8).

\section{Observation Modes}

Due to the conditions and limits discussed in previous sections we suggest following observing modes for X-ray desk on VZLUSAT-1 mission:

(i) Simple imaging: For given parameters (exposure, filtration, binning, and spectroscopic/counting TimePix mode) the instrument would provide single individual data. For each observation one single command is necessary.

(ii) Adrenalin mode: The most important test candidate for optics is the Sun. Therefore several infrared and ultraviolet detectors with wider field of the view are installed to alert the main TimePix detector if some sources with higher values cross the path of the view.

(iii) Monitoring mode: In the meantime when the other instruments and missions onboard VZLUSAT-1 are turned off the X-ray desk may regularly repeat the observations with just one critical parameter (e.g., total sum) influencing whether the data are interesting to be saved or not for later download to the Earth. 
The onboard memory capacity exceeds the uplink and downlink volume estimated for the mean life of the satellite. This monitoring mode can be proceeding even without direct radio connection with ground command station (which is expected twice a day) for a longer period of time. For such a long-term monitoring only one command in memory is necessary (compared to simple imaging mode).

\section{Conclusion}

This article shows the options to test the Lobster eye optics in the space onboard VZLUSAT-1 nanosatellite mission. Astronomical observational candidates are investigated, and estimation of technical settings is presented with necessary laboratory X-ray measurements and analysis.

\section{Competing Interests}

The authors declare that they have no competing interests.

\section{Acknowledgments}

This work was supported by Czech Science Foundation (Grant no. GA14-25251S) and by Grant Agency of the Czech Technical University in Prague (Grant no. SGS13/212/OHK3/ 3T/13).

\section{References}

[1] L. Pina, R. Hudec, A. Inneman et al., "X-ray monitoring for astrophysical applications on Cubesat," in EUV and X-Ray Optics: Synergy between Laboratory and Space IV, R. Hudec and L. Pina, Eds., vol. 9510 of Proceedings of SPIE, SPIE-The International Society for Optical Engineering, Prague, Czech Republic, May 2015.

[2] L. Sveda, R. Hudec, L. Pina, V. Semencova, and A. Inneman, "Lobster eye: technology and imaging properties," in Proceedings of the SPIE EUV and X-Ray Optics: Synergy between Laboratory and Space/Optics+Optoelectronics Symposium, R. Hudec and L. Pina, Eds., vol. 7360 of APR 20-22, SPIE-The International Society for Optical Engineering, Prague, Czech Republic, 2009.

[3] F. Krejci, M. Kroupa, J. Jakubek, P. Bruza, and D. Panek, "Detection of soft X-rays with the pixel detector timepix operated as a highly sensitive dark-current free CCD-like camera," in Proceedings of the IEEE Nuclear Science Symposium and Medical Imaging Conference (NSS/MIC '11), IEEE Nuclear Science Symposium Conference Record/18th International Workshop on RoomTemperature Semiconductor X-Ray and GammaRay Detectors, pp. 1708-1712, IEEE-Institute of Electrical and Electronics Engineers Nuclear \& Plasma Science Society (IEEE NPSS), Valencia, Spain, October 2011.

[4] E. B. Saloman, J. H. Hubbell, and J. H. Scofield, "X-ray attenuation cross sections for energies $100 \mathrm{eV}$ to $100 \mathrm{keV}$ and elements $Z=1$ to $Z=92$," Atomic Data and Nuclear Data Tables, vol. 38, no. 1, pp. 1-196, 1988.

[5] T. Baca, M. Platkevic, J. Jakubek et al., "Miniaturized X-ray telescope for VZLUSAT-1 nanosatellite with Timepix detector," Journal of Instrumentation, vol. 11, 2016.
[6] L. Pina, R. Hudec, V. Tichy et al., "Novel design of a large xray optical system for astrophysical application," in SPIE Optical Engineering + Applications, San Diego, Calif, USA, August 2012.

[7] G. Rottman, “The SORCE mission," Solar Physics, vol. 230, no. 1-2, pp. 7-25, 2005.

[8] B. Aschenbach, "Design, construction, and performance of the rosat high-resolution X-ray mirror assembly," Applied Optics, vol. 27, no. 8, pp. 1404-1413, 1988.

[9] J. H. M. M. Schmitt, S. L. Snowden, B. Aschenbach et al., "A soft X-ray image of the Moon," Nature, vol. 349, no. 6310, pp. 583587, 1991.

[10] J. P. Burrows, M. Weber, M. Buchwitz et al., "The Global Ozone Monitoring Experiment (GOME): mission concept and first scientific results," Journal of the Atmospheric Sciences, vol. 56, no. 2, pp. 151-175, 1999.

[11] M. R. Dobber, "GOME moon measurements, including instrument characterisation and moon albedo," in Proceedings of the 3rd ERS Symposium on Space at the Service of Our Environment, T.-D. Guyenne and D. Danesy, Eds., vol. 414, pp. 743-747, Florence, Italy, March 1997.

[12] F. Jansen, D. Lumb, B. Altieri et al., "XMM-Newton observatory. I. The spacecraft and operations," Astronomy and Astrophysics, vol. 365, no. 1, pp. L1-L6, 2001.

[13] M. G. Watson, A. C. Schröder, D. Fyfe et al., "The XMMNewton serendipitous survey. V. The Second XMM-Newton serendipitous source catalogue," Astronomy and Astrophysics, vol. 493, no. 1, pp. 339-373, 2009.

[14] A. Sandage, P. Osmer, R. Giacconi et al., "On the optical identification of SCO X-1," The Astrophysical Journal, vol. 146, p. 316, 1966.

[15] J. García, J. M. Ramírez, T. R. Kallman et al., "Modeling the oxygen $\mathrm{K}$ absorption in the interstellar medium: an XMMNewton view of Sco X-1," The Astrophysical Journal Letters, vol. 731, article L15, 2011.

[16] C. F. Bradshaw, E. B. Fomalont, and B. J. Geldzahler, "Highresolution parallax measurements of scorpius X-1," The Astrophysical Journal, vol. 512, no. 2, pp. L121-L124, 1999.

[17] S. Sterckx, I. Benhadj, G. Duhoux et al., "The PROBA-V mission: image processing and calibration," International Journal of Remote Sensing, vol. 35, no. 7, pp. 2565-2588, 2014. 

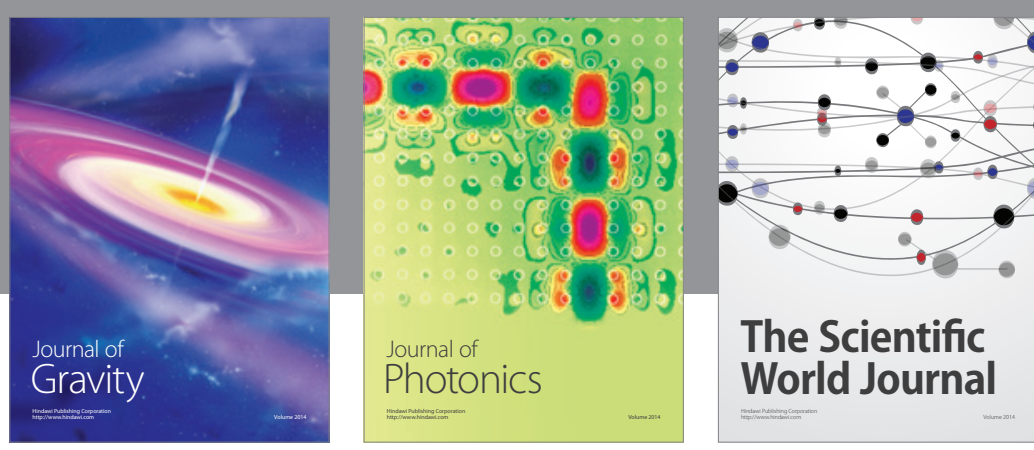

The Scientific World Journal
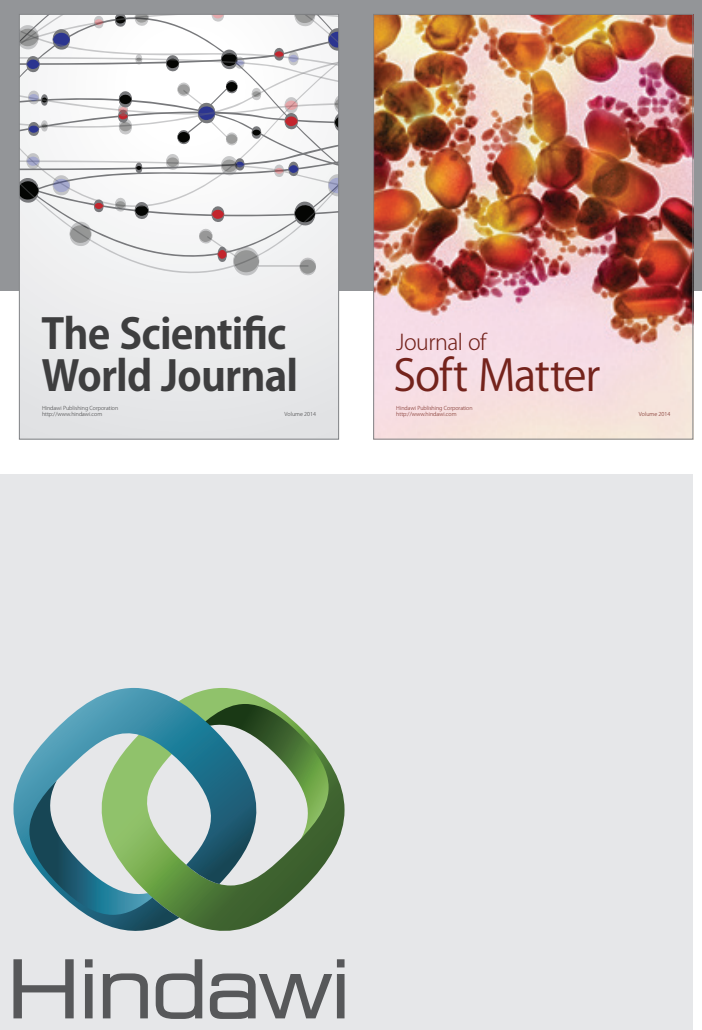

Submit your manuscripts at

https://www.hindawi.com
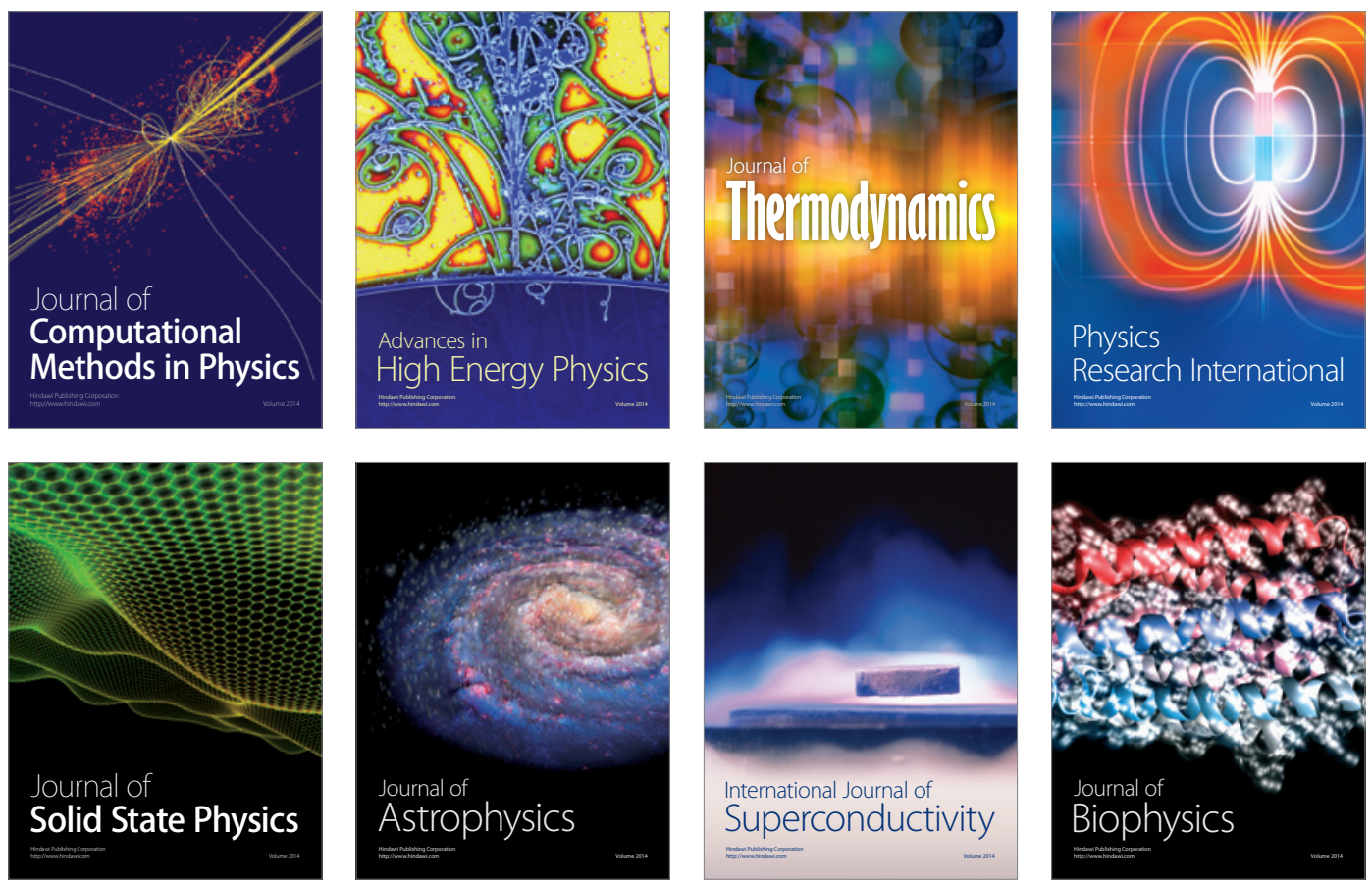
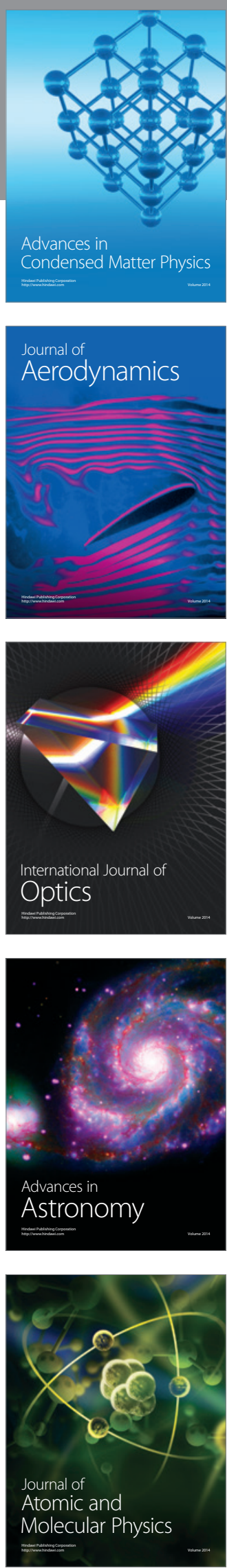